\title{
Theoretical Investigation of Laser Induced Desorption of Small Molecules from Oxide Surfaces: A First Principles Study
}

\author{
T. Klüner* and H.-J. Freund \\ Fritz-Haber-Institut der Max-Planck-Gesellschaft, Faradayweg 4-6, 14195 Berlin, Germany
}

V. Staemmler

Ruhr-Universität Bochum, Lehrstuhl für Theoretische Chemie, Universitätsstraße 150, 44780 Bochum, Germany

R. Kosloff

Department of Physical Chemistry and the Fritz Haber Research Center, The Hebrew University, Jerusalem 91904, Israel

(Received 1 December 1997)

\begin{abstract}
State resolved laser induced desorption of $\mathrm{NO}$ molecules from a $\mathrm{NiO}(100)$ surface is studied theoretically. A full potential energy surface for the excited state was constructed by means of ab initio cluster calculations in addition to the potential energy surface for the ground state. Multidimensional wave packet calculations on these two surfaces allow a detailed simulation of experimental observables, such as velocity distributions and desorption probabilities, on a full $a b$ initio basis. [S0031-9007(98)06374-1]
\end{abstract}

PACS numbers: 79.20.La, 71.10.Li

State resolved laser induced desorption of small molecules from well-characterized surfaces has been the subject of numerous experimental studies in recent years [1], but a detailed understanding even of basic features of final state distributions of the desorbing molecules still remains a challenge. Many theoretical investigations have been reported for the description of such DIET (desorption induced by electronic transitions) processes in which either classical [2,3] or quantum mechanical [4-7] simulations of nuclear motion of the desorbing molecules are presented. All dynamical simulations so far have been empirical because of the lack of sufficiently accurate $a b$ initio potential energy surfaces, especially for the electronically excited states. In this paper, we present a full $a b$ initio potential energy surface (PES) for an excited state involved in a DIET process. As an example, we have studied the system $\mathrm{NO} / \mathrm{NiO}(100)$ for which velocity distributions for different vibrational $\left(v^{\prime \prime}\right)$ and rotational $\left(J^{\prime \prime}\right)$ states of the desorbing NO molecules are shown in Fig. 1 [8]. By performing three-dimensional wave packet calculations on the PESs of the ground and excited electronic states, we can generate the respective distributions.

The method of calculation of the electronically excited states uses a $\mathrm{NiO}_{5}{ }^{8-}$ cluster embedded in a semi-infinite Madelung potential of point charges \pm 2 to simulate the $\mathrm{NiO}(100)$ surface [9]. The NO molecule is adsorbed at an on-top position above a $\mathrm{Ni}^{2+}$ cation. The ground state is characterized by an equilibrium geometry with a tilt angle $\alpha$ of $45^{\circ}$ of the molecular axis with respect to the surface normal. This is in perfect agreement with the experiment [10]. The excited states relevant for laser induced desorption turn out to be charge transfer states of the $\mathrm{NO} / \mathrm{NiO}(100)$ system, where one electron is transferred from the cluster to the NO molecule resulting in an $\mathrm{NO}^{-}$-like intermediate. These states of the adsorbate/ substrate system are calculated by a configuration interaction (CI) calculation in the valence space of the $\mathrm{O} 2 p-$, $\mathrm{Ni} 3 d$-, and NO2 $\pi$-orbitals. Details on the construction of the reference configuration, the generation of the CI space, and selection of a representative excited state are described in Refs. [9,11].

A PES for a representative $\mathrm{NO}^{-}$-like excited state is constructed in which the distance $R$ of the center of mass of the NO molecule and the tilt angle $\alpha$ are varied. The internal N-O distance is kept fixed at the NO gas phase value of 2.175 a.u. [12], because the NO vibration has been found to be decoupled from the translation in the experiments, as illustrated in Fig. 1. This is different for

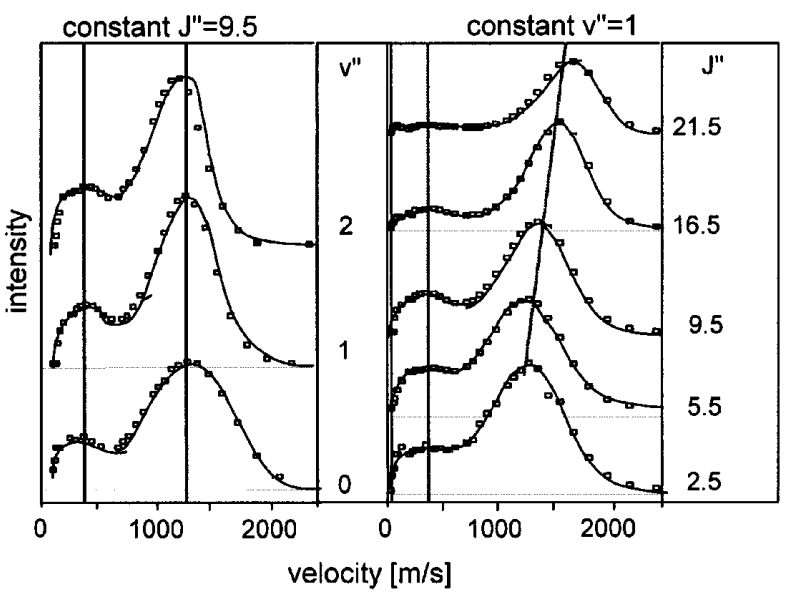

FIG. 1. Experimental velocity distributions of NO molecules after laser induced desorption from $\mathrm{NiO}(100)$, showing a pronounced bimodality. The distributions on the left side indicate a decoupling of translation and vibration. A coupling of rotation and translation is observed in the velocity distributions of the molecules desorbing with high velocities (fast channel, right side) [8]. 
the rotation of the $\mathrm{NO}$ molecule for which a coupling to the translation is found experimentally. Therefore, rotation has to be taken into account in the simulations. The azimuthal corrugation is small for all excited states, but the potentials are strongly anisotropic with respect to the polar angle $\alpha$ [13]. This requires an explicit treatment of this coordinate. We have therefore calculated a twodimensional PES depending on $R$ and $\alpha$. The analytical fit to the pointwise calculated energies is given in Eq. (1) [14] and a graphical representation in Fig. 2.

$$
V(R, \alpha)=-\frac{1}{R}+\sum_{i=0}^{10}\left(\cos ^{i}(\alpha)\left\{\sum_{j=1}^{3} a_{i j} \exp \left[-b_{j}\left(R-R_{0}\right)\right]+\sum_{k=4}^{6} a_{i k} \exp \left[-b_{k}\left(R-R_{0}\right)\right]^{2}\right\}\right) .
$$

Earlier studies have shown that the global shape of the surface can be understood as being due to Coulomb attraction between $\mathrm{NO}^{-}$and the positive hole created within the cluster upon charge transfer and the Pauli repulsion between the $\mathrm{NO}^{-}$and the $\mathrm{O}^{2-}$ anions of the surface [13]. The minimum of the excited state PES is located at a smaller molecule-surface distance than in the ground state (Antoniewicz-like [15] desorption scenario) and the $\mathrm{NO}^{-}$like intermediate prefers an upright position at moderate distances, in contrast to the tilted equilibrium geometry of the electronic ground state [13]. As shown in Fig. 2, the excited state PES possesses a bifurcation which has important consequences for the translational and rotational distribution of the desorbing $\mathrm{NO}$ molecules.

The ground state PES is constructed by adjusting the topology of the potential used in [16] to $a b$ initio results. It is characterized by an equilibrium geometry with $\alpha=$ $45^{\circ}$ and $R=5.5$ a.u., in which the minimum has been scaled to fit the experimental binding energy of $0.52 \mathrm{eV}$ [10]. In Fig. 2, we also indicate the Franck-Condon point at which the wave packet is transferred from the electronic ground state to the excited state potential.

The dynamics of nuclear motion on the two PESs of the ground and the representative excited state is simulated by

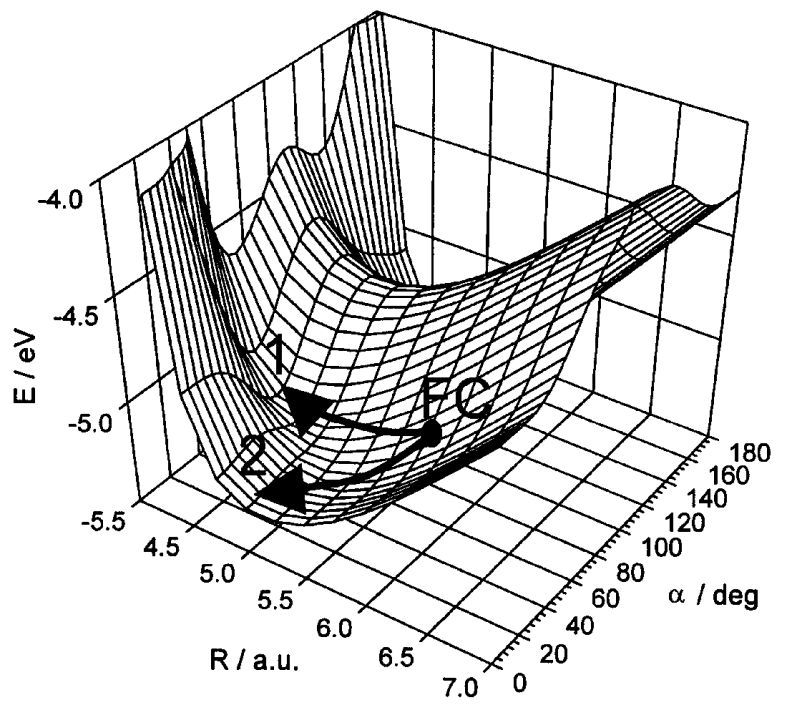

FIG. 2. $\mathrm{NO} / \mathrm{NiO}(100)$ : Charge transfer potential energy surface as a function of the molecule surface distance $R$ and the polar angle $\alpha$. FC denotes the Franck-Condon point. three-dimensional wave packet calculations in which the rotational vibrational ground state wave function of the electronic ground state is taken as the initial wave packet $\psi_{0}$ for a propagation on the ab initio excited electronic state PES starting from the Franck-Condon point FC. The coordinates of the wave packets were chosen as the molecule-surface distance $R$, the polar angle $\alpha$, and the azimuthal angle $\varphi$. The desorption scenario is simulated by transferring the wave packet after propagating it for a residence lifetime $\tau_{R}$ on the excited state potential onto the electronic ground state PES. The wave packet is then propagated under the influence of the ground state PES, and the desorbing part of the wave packet is analyzed after a time $t_{g}$. In all calculations, the Chebychev polynomial expansion of the time evolution operator proposed by Kosloff has been used [17].

Velocity distributions are obtained as momentum space probability densities in the asymptotic region of the ground state PES. The propagation in potential free space is performed as a phase shift of the wave function in momentum space according to Heather and Metiu [18]. The incoherent average scheme for different residence lifetimes $\tau_{R}$ of $N$ quantum trajectories proposed by Gadzuk [19] is applied. The only parameter in this average scheme is the resonance lifetime $\tau$. In order to simulate the experimentally observed state resolved velocity distributions of the desorbing NO molecules, a resonance lifetime $\tau$ of $24.19 \mathrm{fs}$ (1000 a.u.) has been selected. This yields a desorption probability of $P_{\mathrm{des}}=3.3 \%$ (square-norm of the wave packet in the asymptotic region) which is in good agreement with experimental results for oxide systems [8]. It has to be pointed out that this adjustment of the desorption probability to experimental values by selecting the resonance lifetime is the only empirical input in the simulation of the dynamics of nuclear motion. A full $a b$ initio treatment of the relaxation process would require the calculation of nonadiabatic coupling elements which is beyond the scope of this study.

Using this resonance lifetime, velocity distributions are obtained for selected rotational quantum numbers (Fig. 3). It is clearly seen that the main experimental features as shown in Fig. 1 are reproduced. The velocity distributions are in the correct velocity range and exhibit the experimentally observed bimodality. Furthermore, the coupling of rotation and translation in the velocity distributions is also reproduced. Even details of the experimental observations 


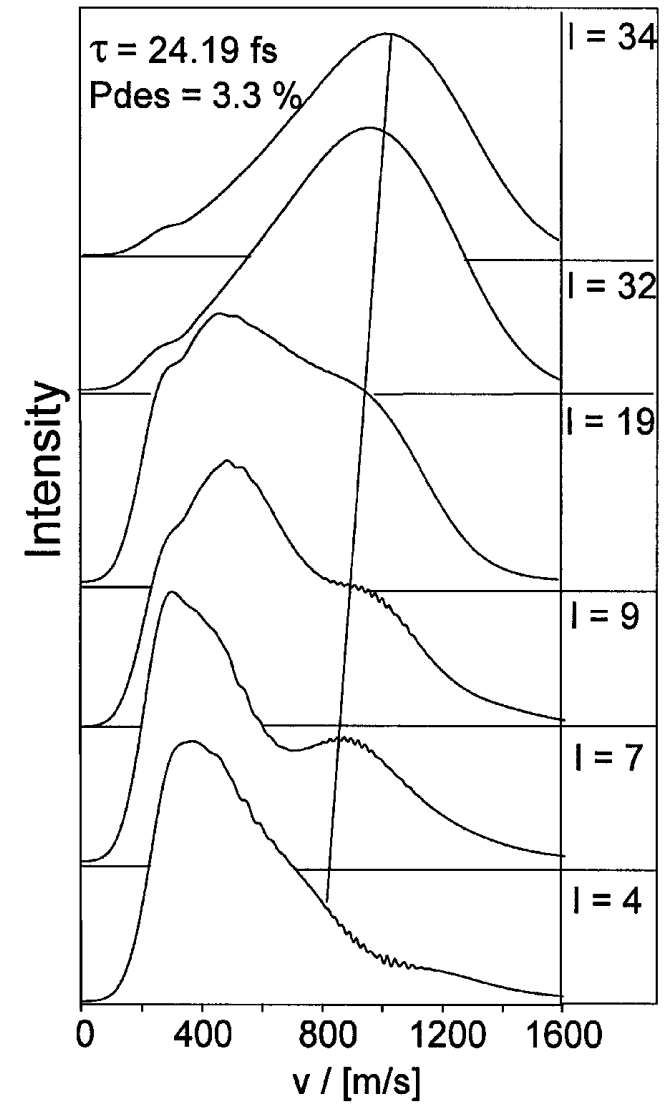

FIG. 3. Velocity distributions for a resonance lifetime of 24.19 fs for different rotational quantum numbers $l$.

can be understood through the analysis of the time evolution of the initial wave packet on the excited state PES. Because of the bifurcation shown in Fig. 2, two pathways exist on the excited state PES which different partial wave packets can take. Pathway 1 results in wave packets taking a way along the valley of the potential. This leads to an accumulation of a large amount of kinetic energy and short molecule-surface distances before the relaxation onto the ground state PES occurs. On the other hand, pathway 2 results in an early deacceleration of the wave packet, relaxation at large distances $R$, and slowly desorbing particles. Thus, the bimodality of velocity distributions is the result of a bifurcation of the wave packet due to the topology of the excited state PES. This conclusion has been proven by a thorough analysis of the wave packet dynamics [11]. As one example, the velocity distribution after a residence lifetime of $\tau_{R}=52 \mathrm{fs}$ in the excited state is presented in Fig. 4 to demonstrate these features.

The crucial role of the topology of the PES emphasizes the essential need for the $a b$ initio calculation of excited state PES to allow a realistic simulation of nuclear motion. It seems very unlikely that any empirical estimate of an excited state PES would have guessed a topology similar to that of our PES, which is important even for a qualitative understanding of experimental results.

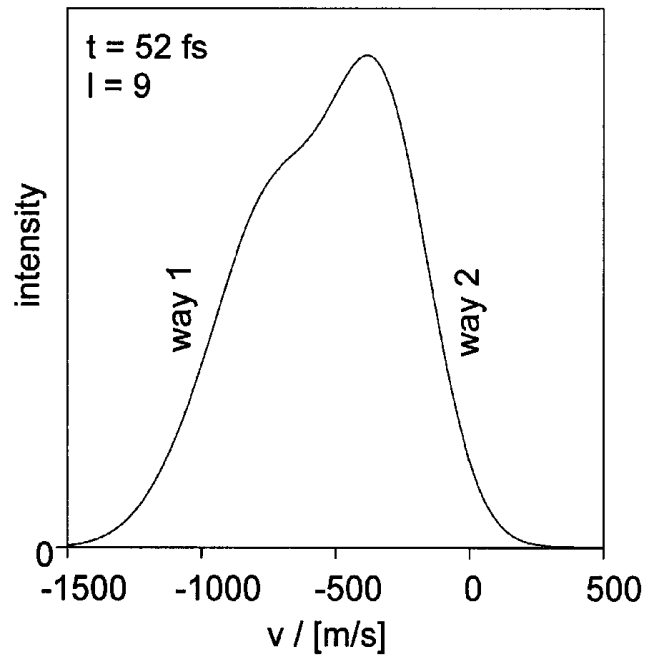

FIG. 4. Velocity distribution for a residence lifetime of $52 \mathrm{fs}$ in the excited state for an angular momentum quantum number of $l=9$.

The quantitative analysis of the final state distributions turns out to be more complicated because of the influence of the ground state potential on the nuclear motion after the relaxation. Detailed studies will be published elsewhere [11]; however, the ground state PES does not affect the main results presented in this paper as far as the origin of bimodality is concerned.

In conclusion, we presented the first three-dimensional wave packet study for a DIET process using nonempirical $a b$ initio potential energy surfaces. It has been shown that even details of the observed final state distributions can be quantitatively reproduced. A new mechanistic understanding of experimental results, in particular the origin of bimodal velocity distributions, has been achieved.

Financial support by the German-Israeli Foundation (GIF), the Studienstiftung des deutschen Volkes, and the Deutsche Forschungsgemeinschaft (DFG) are gratefully acknowledged.

*Corresponding author.

[1] F. M. Zimmermann and W. Ho, Surf. Sci. Rep. 22, 127 (1995).

[2] D. Menzel and R. Gomer, J. Chem. Phys. 41, 3311 (1964); P. A. Redhead, Can. J. Phys. 42, 886 (1964).

[3] E. Hasselbrink, Chem. Phys. Lett. 170, 329 (1990).

[4] W. Brenig, Z. Phys. B 23, 361 (1976).

[5] Z.W. Gortel and A. Wierzbicki, Phys. Rev. B 43, 7487 (1991).

[6] P. Saalfrank, Chem. Phys. 193, 119 (1995).

[7] A. R. Burns, E. B. Stechel, and D. R. Jennison, Phys. Rev. Lett. 58, 250 (1987).

[8] T. Mull, B. Baumeister, M. Menges, H.-J. Freund, D. Weide, C. Fischer, and P. Andresen, J. Chem. Phys. 96, 7108 (1992).

[9] T. Klüner, J. Freitag, H.-J. Freund, and V. Staemmler, J. Chem. Phys. 104, 10030 (1996). 
[10] H. Kuhlenbeck, G. Odörfer, R. Jaeger, G. Illing, M. Menges, T. Mull, H.-J. Freund, M. Pöhlchen, V. Staemmler, S. Witzel, C. Scharfschwerdt, K. Wennemann, T. Liedtke, and M. Neumann, Phys. Rev. B 43, 1969 (1991).

[11] T. Klüner, S. Thiel, H.-J. Freund, V. Staemmler, and R. Kosloff (to be published).

[12] K.P. Huber and G. Herzberg, Constants of Diatomic Molecules (Van Nostrand Reinhold, New York, 1979).

[13] T. Klüner, J. Freitag, H.-J. Freund, and V. Staemmler, J. Mol. Catal. A 119, 155 (1997).
[14] The parameters will be distributed to interested readers on request.

[15] P. R. Antoniewicz, Phys. Rev. B 21, 3811 (1980).

[16] B. Baumeister and H.-J. Freund, J. Phys. Chem. 98, 11962 (1994).

[17] R. Kosloff, J. Phys. Chem. 92, 2087 (1988).

[18] R. Heather and H. Metiu, J. Chem. Phys. 86, 5009 (1987).

[19] J. W. Gadzuk, Surf. Sci. 342, 345 (1995). 